\title{
ENTREVISTA A NEIL MACCORMICK
}

Manuel Atienza

Universidad de Alicante

\begin{abstract}
Manuel Atienza: Para empezar, me gustaría conocer algunos datos autobiográficos. ¿Dónde estudiaste Derecho?

Neil MacCormick: Fui a la Universidad de Glasgow desde 1959 a 1963 y me gradué como M.A. con honores en filosofía y en literatura. Gané entonces una beca para continuar mis estudios, entre 1963 y 1965, en el Balliol College, en la Universidad de Oxford, donde obtuve una graduación en Derecho (B.A. en Jurisprudence).
\end{abstract}

\section{M.A.: ¿Cómo eran entonces las universidades inglesas (o británicas)?}

N.M.: La filosofía en las universidades escocesas, particularmente en Glasgow, no estaba aún bajo la influencia de «la filosofía del lenguaje ordinario». Mi interés en la filosofía práctica se centraba particularmente en las ideas neokantianas de W. D. LAMONT, en Deontología tal y como la defendía W. G. MaClagan y W. D. Ross, y en los moralistas británicos del XVIII como Hume, Butler, Adam Smith y Thomas ReID. También empecé a leer la filosofía moral de R. M. HARE, que conectaba bien con mis intereses kantianos, y encontré los escritos de J. L. Austin sobre Sense and Sensibilia y otros temas enormemente estimulantes. Estábamos expuestos también a ciertas dosis de WitTGENSTEIN, pero nunca me sentí atraído por su manera de escribir y de pensar. También existía un considerable interés hacia John Stuart MiLl y el utilitarismo inglés.

En Oxford, aprendí mucho de Derecho inglés y de Derecho romano, pero en particular recibí la influencia de H. L. A. HART, entonces catedrático de Jurisprudence. Fui a sus clases (sobre derechos y deberes y sobre KELSEN) y leí El concepto de Derecho. Por entonces, mi intención era volver a Escocia y dedicarme al ejercicio del Derecho en Edimburgo, de manera que cuando se me ofreció un puesto de profesor en $\mathrm{St}$ Andrews University (en el Queen's College, en Dundee) lo acepté como un medio para un fin.

M.A.: ¿Han cambiado mucho las universidades británicas en las últimas décadas?

N.M.: Las universidades se han hecho más grandes y han tratado de volverse más inclusivas socialmente. La organización de la enseñanza está más altamente formalizada de lo que solía, pero todavía hay un buen contacto entre el staff de los profesores, incluidos los catedráticos seniors, y los estudiantes de grado. Todos tenemos ahora ordenadores y esto transforma mucho la vida de un profesor. El gobierno nos obliga a mirar demasiado hacia objetivos vinculados con una investigación productiva, lo que supone demasiado esfuerzo mecánico. Pero, en su conjunto, las cosas que han cambiado, han cambiado, en mi opinión, básicamente para mejor. 


\section{M.A.: ¿Quiénes fueron tus maestros?}

N.M.: William LAmONT, profesor adjunto de filosofía moral, en la Universidad de Glasgow; Robin DownIE, posteriormente profesor de filosofía moral en la Universidad de Glasgow; J. J. RusSELL, posteriormente profesor de filosofía en la Universidad de Kansas Howard Horsburgh. Después, en Oxford: D. R. HARRIS (posteriormente director del Center for Socio-Legal Studies, en la universidad de Oxford); W. A. J. [Alan] Watson, y H. L. A. HART.

\section{M.A.: ¿Por qué te interesaste en la filosofía del Derecho?}

N.M.: Porque estaba fascinado con la filosofía, pero quería dedicarme a la práctica del Derecho. Cuando se me presentaron en el camino oportunidades para lograr un puesto académico, primero en Dundee, luego en Oxford y finalmente la Regius Chair en Edimburgo, decidí que la filosofía del Derecho era, después de todo, la vocación de mi vida. Nunca lo he lamentado.

\section{M.A.: Me gustaría saber más detalles de tu relación con HART.}

N.M.: Cuando obtuve el puesto de Fellow del Balliol College, en 1967, lo veía con cierto temor reverencial, como un gigante de mi campo de estudios, cuya obra encontraba muy convincente. Había ido a sus clases, pero nunca había tenido una relación personal con él. Me dispensó una bienvenida amistosa, como colega más joven dedicado también a la filosofía del Derecho, y a su debido tiempo trabajé con él en algunas cuestiones referidas a la Oxford Union Society, de la que él era uno de los administradores y yo fui durante cierto tiempo tesorero suplente.

Con John FInNIS y Richard BUXTON fui el responsable de un seminario de posgrado (dirigido a candidatos para el BCL y también para estudiantes de doctorado) sobre algunos textos seleccionados de teoría general del Derecho. HART asistía a ellos regularmente, como en su momento hicieron Joseph RAZ y Ronald DWORKIN. HART se retiró de su cátedra de teoría general del Derecho (Jurisprudence) muy al comienzo de mi estancia en Oxford, y el nombramiento, bastante sorprendente, de Ronald DwORKIN tuvo lugar al final de mi primer año.

En la habitación de HART en el University College, y luego en la de Jonh FinNIS y más tarde en la de Ronald DwORKIN, se reunía un grupo de discusión que incluía a todos los anteriormente nombrados más Gareth Evans, Brian SIMPSON, David BENTLEY, Philip LEWIS, Jean FLOUd y ocasionalmente visitantes como Adam PODGORECKI, Philippe Nonet, Philip SELzNick y Harry Frankfurt. Mantuve la conexión después de mi marcha a Edimburgo, en 1972, pero inevitablemente el contacto se fue debilitando con el tiempo.

Escribí mi propio libro sobre HART en Edimburgo, en 1979-80, y se publicó en 1981 como el primero de la colección "Juristas" editada por William TwINING. Era un intento de exponer y valorar de manera breve sus contribuciones académicas, consideradas a la luz de las informaciones biográficas básicas que parecían necesarias para poner la obra jurídica en su contexto.

Hasta entonces, la mayor parte de mis propios escritos y de mis enseñanzas habían tenido lugar dentro del paradigma hartiano, y mi conclusión en el libro consistía en 
gran medida en un amplio acuerdo con la obra de HART, tal y como yo la interpretaba. Posteriormente, resultó claro que mi interpretación situaba a HART mucho más cerca de la tradición del "Derecho natural" de lo que él deseaba. Sus últimos años y el postcripto póstumo a El Concepto de Derecho (en la segunda edición) estuvieron marcados por una adhesión aún más firme que antes al positivismo inclusivo.

Al libro sobre "HART" le había precedido mi Legal Reasoning and Legal Theory, de 1978, en el que yo, en efecto, trataba de presentar una teoría comprehensiva del razonamiento jurídico que en parte estaba basada en, y que en todos sus aspectos era compatible con, la teoría general del Derecho de El Concepto de Derecho. Se publicó en la colección Clarendon que editaba HART, y él me hizo comentarios editoriales muy útiles.

En los primeros años ochenta yo era considerado, de manera correcta, como un miembro de la misma escuela de pensamiento que HART, de su círculo, aunque con una cierta desviación hacia el reconocimiento de una mayor interpenetración mutua entre el Derecho y la moral, de lo que HART consideraba correcto. A lo largo de todo este tiempo de trabajo hice amistad personal tanto con Herbert HART como (en menor medida) con su mujer Jenifer, pero nunca fuimos amigos íntimos y (por ejemplo) nunca fui uno de los que se juntaban con los HarT en su casa de campo en Cornwall.

Como intelectual, era bastante austero y muy crítico al enjuiciar lo que consideraba descuidado o poco reflexivo. A veces me parecía demasiado precipitado en sus juicios y no inclinado a ver la fuerza de los puntos de vista con los que discrepaba. Era un maravilloso y bondadoso, aunque riguroso, crítico de las obras en preparación de los colegas. Pensaba lo suficientemente bien de mí como para apoyar mi nombramiento para la cátedra de Edimburgo en 1972, aunque todavía sólo tenía 31 años de edad. Era una persona bastante tímida, pero afable y amable en el trato con los que eran miembros de su - amplio- círculo de colegas amigos en Oxford y en otros lados. Tenía un ingenio seco y podía ser divertido de una manera acre.

M.A.: ¿Qué te parece la reciente biografía sobre HART de Nicola LACEY? ¿Te ba hecho cambiar de opinión sobre HART en algún sentido?

N.M.: Admiro el libro de LACEY. Al tiempo que desvela muchos aspectos de su vida privada y de sus ideas que no conocía, confirma más bien que rectifica mi imagen general sobre él y no ha hecho que disminuya mi admiración tanto por HERBERT como por Jenifer HART, sino que en todo caso la ha aumentado. Para mi alivio, he encontrado que el boceto biográfico de mi libro ha resultado ser ampliamente acertado, incluso a la luz de la biografía mucho más penetrante realizada por LACEY.

M.A.: ¿Cuáles son, en tu opinión, los puntos más fuertes y más débiles de la obra de HART?

N.M.: Acabo de terminar una obra mayor titulada Instituciones del Derecho que fija mis posiciones (probablemente) finales en teoría general del Derecho emprendidas desde una perspectiva interpretativo-analítica y prestando la atención debida a los recientes avances en los estudios sociojurídicos. Es, dicho brevemente, mi versión de la teoría institucional del Derecho que WEINBERGER y yo comenzamos a proponer en los ochenta. A la luz de ello, diría lo siguiente: 
El aspecto en el que la obra de HART resulta más iluminadora y perdurable se refiere a la necesidad de entender la conducta gobernada por reglas desde el "punto de vista interno". Esto es esencial para desarrollar una teoría clara y convincente de las normas; pero las reglas son sólo un tipo de normas. El análisis del Derecho como la unión de reglas primarias y secundarias, aunque lleno de intuiciones valiosas, es, al final, incompleto e insatisfactorio. Se necesita un nuevo comienzo. Una versión de una "teoría de la norma básica" es más satisfactoria que una teoría de una "regla de reconocimiento" para explicar la unidad de un sistema jurídico en el contexto de un Estado constitucional (Rechsstaat, Estado de Derecho). Las instituciones jurídicas se conectan con la política y la economía y tienen carácter fundamental para el Estado y para la sociedad civil. El Derecho penal es una parte esencial de los fundamentos de la paz social y por tanto de la sociedad civil. Todo esto le aleja a uno bastante de la concepción hartiana del Derecho. El Derecho y la moral son de hecho conceptualmente distintos, pero sigue siendo cierto que elementos mínimos de respeto por la justicia son esenciales para el reconocimiento de que un orden normativo tiene carácter "jurídico".

M.A.: Antes de entrar a comentar algunos aspectos particulares de tu obra, me gustaría que presentaras un panorama general de ella. ¿Podrías señalar cuáles son las aportaciones que consideras de mayor importancia y las circunstancias en que surgieron? ¿Qué evolución ha habido en tu concepción de la teoría del Derecho, después de más de 30 años de actividad intensa?

N.M.: Mi educación filosófica en Glasgow dejó en mí un interés por cuestiones de lógica y de razonamiento informal. En mi primer nombramiento académico, en 1965-67, en el Queen's College de Dundee (en la Universidad de St. Andrews), mi director allí, el profesor Iam WiLLOCK, me pidió que preparase un curso de conferencias sobre lógica y razonamiento jurídico. Conectado con esto hubo un trabajo muy temprano, «Can Stare Decisis be Abolished?» (1966, Juridical Review 1967). Continué con este interés cuando en 1967 me trasladé a Oxford y, durante los años 1967-72, tuve una controversia con Ronald DwORKIN, en la que asumía la posición de H. L. A. HART en las disputas de entonces acerca del lugar de los principios en el Derecho y en el razonamiento jurídico. El resultado final de ello fue mi Legal Reasoning and Legal Theory (Oxford: Oxford University Press, 1978, 2. a ed., 1994).

En Oxford, y de nuevo estimulado por intereses que habían surgido en Glasgow, hice algún trabajo en la teoría de los actos de habla en direcciones que me parecían de considerable importancia para el Derecho y los conceptos jurídicos, todavía estrechamente conectado con mi interés en la obra de HART. Dos contribuciones que muestran este interés son «Voluntary Obligations and Normative Powers» (1972, XLVI, Aristotelian Supplementary Volume, pp. 59-78) y «Legal Obligation and the Imperative Fallacy», en Oxford Essays in Jurisprudente, 2nd Series [editado por A. W. B. SIMPSON, Oxford Clarendon Press, 1973, pp. 100-130). Ello se conecta directamente con mi teoría de los actos institucionales y por tanto con el tema de mi lección inaugural como profesor en la Universidad de Edimburgo, «Law as institucional» (1973: Edimburgo: Edinburgh University Inaugural Lectura, n. ${ }^{\circ}$ 52; también publicado en Law Quaterly Rev 70 (1974) 102-129).

Durante mis primeros años en Edimburgo estuve muy dedicado a desarrollar un balance completo del razonamiento jurídico. El trabajo principal, antes mencionado, 
se publicó en 1978, pero no antes de que hubiese tenido un encuentro con Chaim PERELMAN en una conferencia en Stirling, en 1976, que tuvo una considerable influencia en mi pensamiento y me llevó luego a algunos trabajos publicados por invitación suya ${ }^{1}$.

En 1979, en el congreso mundial de la IVR, en Basilea, participé en la misma sesión plenaria que Ota WEINBERGER, de Graz, presentando un trabajo, «On Analytical Jurisprudence», en el que recogía un tema de Peter HACKER relativo al aspecto "hermenéutico" del pensamiento de HART sobre la naturaleza de la conducta guiada por reglas y su "aspecto interno". El encuentro con WeINBERGER llevó a un reconocimiento mutuo de la semejanza de nuestras aproximaciones al carácter "institucional" del Derecho. Esta interacción continuó luego en el mismo año en una conferencia organizada por Aulis AARNIO en Finlandia, más tarde en Edimburgo en una reunión sobre KELSEN de la ALSP, en 1981, y en el congreso mundial de la IVR en Helsinki, en 1983. Todo ello llevó finalmente a la edición conjunta de una serie de ensayos, traducidos al alemán (los míos) y al inglés (los de WeINBERGER), publicados como Grundlagen des institutionalistischen Rechtspositivismus (1986) у como Institucional Theory of Law (1987).

Otros encuentros significativos en 1979, en el Congreso Mundial, fueron con Robert AleXY y con Aleksander PECZENIK, junto con Aulis AARNIO. Durante una visita a la Universidad de Saarland en la primavera de 1979, emprendida con el propósito de remediar mi ignorancia del alemán, había descubierto la Theorie der juristischen Argumentation de AleXY y el Reasoning on Legal Reasoning de AARNIO. La obra de PECZENIK sobre Causes and Damages la descubrí sólo durante el Congreso de Basilea. Resultaba claro para mí, y para mis colegas, que los cuatro convergíamos en cuanto a nuestros intereses acerca del razonamiento jurídico, al tiempo que mostrábamos algunas diferencias características atribuibles a las tradiciones filosóficas de las que procedíamos. En su momento escribí el que creo fue el primer trabajo en inglés que llamaba la atención sobre la obra de ALEXY ${ }^{2}$ y sobre su importancia, y posteriormente mi antigua estudiante de doctorado (y querida amiga) Ruth ADLER y yo preparamos una traducción al inglés de la Theory of Legal Argumentation (1988); nuestra capacidad para colaborar en traducciones había quedado ya establecida con el trabajo que ya habíamos hecho en las traducciones WEINBERGER/MACCORMICK.

En 1980 apareció el libro de John FinNis Natural Law and Natural rights. Como amigo de John FINNIS, había leído el texto original y hecho algunas sugerencias menores antes de la publicación. Esa obra me causó una gran impresión y me llevó a volver a valorar por completo mi comprensión del "Derecho natural", lo que se ve confirmado en diversos trabajos posteriores ${ }^{3}$. Por ese tiempo también, instigado por Willam TwiNING, me había comprometido a escribir el primer volumen de una serie de estu-

1 «Formal Justice and the Form of Legal Arguments», 1976, VI Études de Logique Juridique, 103-118; «The Motivation of Judgement in the Common Law», La motivation des Décisions de Justice, ed. por Ch. Perelman y Foriers, 1978, Bruselas: Brylant, pp. 167-94; «On Reasonableness», en Les Notions à Contenu Variable en Droit, ed. por Ch. Perelman, et al., 1984, Bruselas: Bruylant, pp. 233-48.

2 «Legal Reasoning and Practical Reason», Midwest Studies in Philosphy VII (ed. por French, et. al., Ninneapolis, 1982), pp. 271-286.

3 Véase en particular «Natural Law and the Separation of Law and Morals», en George, R. P. (ed.), 1992: Natural Law Theory (Oxford: Clarendon Press, pp. 105-33). 
dios breves y en parte biográficos de juristas importantes — la serie "Jurists" - . Mi H. L. A. Hart se publicó en la primavera de 1981.

Éste ha sido un balance muy bien recibido de los principales elementos de la filosofía jurídica de HART. Como te decía, incluso después de la reciente publicación de la biografía de altos vuelos de Nicola LACEY, H. L. A. Hart: The Nibtmare and the Noble Dream (2005), parece que mi libro sobre HART es un balance ampliamente aceptado de la teoría jurídica hartiana. Sin embargo, tal vez bajo la influencia de FinNIS, pero también en direcciones vinculadas con mi formación filosófica escocesa, hizo parecer a HART más iusnaturalista de lo que él (HART) deseaba, como una vez le dijo a William Twining. En su momento, el nuevo Prefacio a la segunda edición de The Concept of Law que se publicó póstumamente mostró cómo HaRT había fortalecido la línea positivista en su propio pensamiento. Esto iba contra las ideas que yo había sugerido como la línea más atractiva para desarrollar ciertas ideas principales en The Concept of Law. Así, la publicación de H. L. A. Hart marcó la línea divisoria de mi periodo fuertemente "hartiano" como jurista. Sin despreciar para nada la enorme influencia que su pensamiento y personalidad tuvo sobre mí, empecé desde entonces a desarrollar líneas de pensamiento que divergían cada vez más ampliamente del pensamiento de HART y desde luego de su último pensamiento.

En los últimos años ochenta y en los noventa, mi trabajo sobre razonamiento jurídico, en colaboración con mi íntimo colega y amigo Zenon BANKOWSKI, se concentró cada vez en forma más estrecha en problemas de interpretación considerados de manera comparativa. Eso se desarrolló a través del trabajo del grupo de profesores reunidos inicialmente a iniciativa de Bob Summers, de la Univesidad de Cornell, y más tarde organizado conjuntamente por Bob y por mí, con una considerable aportación de ZENON. El llamado "Bielefelder Kreis" (un nombre que derivaba de diversas sesiones de trabajo que tuvieron lugar en el ZiF en Bielefeld a iniciativa de Ralf DreIER) llevaron a la producción de dos interesantes series de ensayos sobre Interpreting Statutes: A Comparative Study (1991) e Interpreting Precedents: A Comparative Study (1997) (editados por SUMMERS y MACCORMICK).

BANKOWSKI y yo organizamos conjuntamente el Congreso Mundial de la IVR en Edimburgo, en 1989, sobre el tema «llustración, derechos y revoluciones», y coeditamos un libro con las ponencias de las sesiones plenarias con el mismo título («Enlightment, Rights and Revolutions») y después del Congreso actuamos como editores principales de una serie de publicaciones sobre temas conexos. En parte era una cuestión de deber hacia los colegas de la IVR, pero expresaba también un interés que había tenido durante mucho tiempo, del que dan prueba muchos escritos, por el pensamiento jurídico, moral y político de la ilustración escocesa, considerado a la luz de sus amplias conexiones internacionales.

Debido en parte a compromisos políticos fuera del mundo académico, en los primeros noventa estaba sumamente interesado en desarrollar ideas acerca de cómo la emergencia y el desarrollo del "nuevo orden jurídico" de las Comunidades Europeas (y ahora de la Unión Europea) desafiaban muchas categorías tradicionales de la teoría del Derecho; de manera muy notable, la de "soberanía". Mi conferencia Chorley de 1992, «Beyond the Sovereign State» (56 Modern Law Review, 1-18, 1993) ha resultado ser un punto de partida bastante influyente. En su momento fue seguida por la 
publicación de Questioning Sovereignity: Law, State and Nation in the European Commonwelth (1999) que incluyó versiones revisadas de diversos trabajos publicados en los años intermedios sobre temas conexos. También incluye el más maduro de mis escritos sobre las ideas de nación y de nacionalismo y la posibilidad del nacionalismo cívico. Entre 1999 y 2003 fui miembro, por Escocia, del Parlamento Europeo y eso incluyó dos años como miembro alterno de la "Convención para el futuro de Europa" presidida por el anterior presidente Giscard d'Estaing, que produjo el borrador de Tratado que establecía una Constitución para Europa. Para mí fue una experiencia práctica de lo más excitante y que conectaba con mi anterior trabajo teórico, sobre el que escribí un breve texto: Who's Afraid of a European Constitution? (Exeter: Imprint Academia, 2005).

En 1997, después de un largo periodo en el que tuve tanto que dedicarme a pesados deberes administrativos en la Universidad de Edimburgo como emplear una gran parte de mi tiempo sobrante en actividades políticas, fui tan afortunado como para obtener un Research Professorship del Leverhulme Trust. Eso me permitió dedicar no menos del 80 por 100 de mi tiempo de trabajo a investigar y a escribir, a reunir los diversos cabos de trabajo iusfilosófico en los que había estado ocupado durante los años ochenta y noventa. Mi proyecto se tituló «Derecho, Estado y razón práctica», y preveía la publicación de cuatro libros. Questioning Sovereignty fue el primero de ellos. Durante mis cinco años en el Parlamento Europeo sólo pude hacer pequeños progresos, pero el Leverhulme Trust muy generosamente renovó mi beca cuando abandoné el Parlamento en junio de 2004. Desde entonces, Rhetoric and the Rule of Law: A Theory of Legal Reasoning (Oxford: Oxford University Press, 2005) ha actualizado completamente mi teoría acerca del razonamiento jurídico. Este libro muestra una evolución hacia bases compartidas con Ronald DwORKIN sobre el problema de "respuestas correctas" objetivas a cuestiones jurídicas disputadas. Próximamente, en enero de 2007, Institutions of Law: An Essay in Legal Theory (Oxford: Oxford University Press, 2007) actualiza del todo mi versión de la teoría institucional del Derecho. Lo hace en una forma declaradamente postpositivista, desarrollando una aproximación interpretativa a la teoría del Derecho analítica que está completamente en armonía con algunos desarrollos recientes e importantes de la sociología del Derecho y de los estudios socio-jurídicos de carácter más general.

Mi objetivo último es un libro acerca de la razón práctica en la moral y en el Derecho que se publicará hacia mediados del 2009. En él exploraré de una manera completa la posibilidad de la ética discursiva como base para desarrollar objetivamente principios morales que puedan ponerse a prueba y daré cuenta de manera fidedigna de la centralidad de la autonomía o autocontrol para la acción moral y el pensamiento moral. Procuraré analizar la conexión de estos resultados con posiciones bien conocidas de la teoría clásica del Derecho natural. En sus conclusiones, este libro unirá y reconciliará mi aproximación al razonamiento jurídico y mi teoría institucional del Derecho de carácter postpositivista, así como la teoría de la soberanía y del Estado.

\section{M.A.: ¿Cabe entonces hablar de estadios o de etapas en tu obra?}

N.M.: Reducido a estadios, yo diría que los años 1965-81 fue para mí un periodo en el que estuve estrechísimamente vinculado a la obra de HART, como seguidor de la misma, aunque con conexiones independientes que derivaban de otras influencias. 
1981-1995 fue un periodo de reconsideración de temas centrales y de firme distanciamiento de HART, especialmente, del último HART. Desde 1995 he efectuado un balance global de mi propio pensamiento maduro, centrado en el institucionalismo postpositivista desarrollado en Institutions of Law y que ha de ser reforzado finalmente por mi libro sobre la razón práctica en la moral y en el Derecho.

M.A.: Verdaderamente es un curriculo impresionante que me recuerda, en muchos aspectos, al de NINO. Él también estuvo intensamente implicado en política y defendió una concepción amplia del Derecho dirigida a evitar su aislamiento (tanto del Derecho como de la ciencia jurídica) en relación con otros elementos de la razón práctica. Me parece también que, en cuanto a los aspectos de contenido, vuestras obras son muy similares.

Permite que te formule una pregunta sobre tus ideas politicas. En tu anterior respuesta mencionabas la posibilidad de un "nacionalismo cívico". Como sabes, el nacionalismo está en el centro del debate político en España desde hace muchos años, y mucha gente (incluyendo un cierto número de filósofos del Derecho, de la moral y de la politica) piensa que la conexión entre civismo y nacionalismo es imposible o, cuando menos, muy problemática. Los principios de la ética de NINO tampoco resultan - me parecefácilmente compatibles con el nacionalismo. Podrías explicar brevemente cuál es tu idea del nacionalismo cívico.

N.M.: La respuesta a esa pregunta está desarrollada en el capítulo 11 de mi libro Questioning Sovereignty. Lo esencial de la misma se encuentra en varios párrafos de las pp. 170 a 183 , que te transcribo:

«Para [la idea de "nacionalismo liberal"] la idea de nacionalismo cívico es fuertemente material. El "nacionalismo cívico" identifica la nación en términos de las lealtades compartidas de sus miembros hacia ciertas instituciones cívicas. Éstas se entienden en términos amplios para incluir, por ejemplo, normas e instituciones jurídicas, órganos políticos representativos, ramas de la administración pública y local, la organización de la educación, iglesias y comunidades religiosas en sus aspectos seculares y otras instituciones semejantes que tengan una localización supuestamente territorial a las que referirse. Aquí son relevantes tanto instituciones de la sociedad civil como del Estado. Las instituciones cívicas localizadas territorialmente pueden ser objeto de lealtad, entendidas como "nuestras" por la gente entre la que desarrolla sus funciones. Como instituciones cívicas tienen, necesariamente, una gran significación política para la comunidad a la que, en cierta medida, definen.

Naturalmente, es posible, y quizás deseable, para tales instituciones cívicas extenderse hasta incluir una constitución y toda la panoplia de lo estatal. Tal vez sin ello la cualidad cívica de las instituciones cívicas sea demasiado precaria, pero sería un error requerir eso por definición, pues hacerlo así es simplemente confirmar la asunción en principio dudosa de que los Estados que actualmente existen comprenden también la totalidad de las naciones, en cualquier caso, la totalidad de las naciones que pueden ser comprendidas en el sentido cívico. Con independencia de que la nación cívica sea o tenga un Estado o, a fortiori, un Estado soberano independiente, la clave de la idea de una nación cívica es que ella está en principio abierta a una pertenencia voluntaria. La comunidad definida por lealtad a las instituciones está abierta a cualquiera que elija vivir en el territorio y dar su lealtad a las instituciones. Cambiarse a otro lugar y 
a otras lealtades es también posible y no una traición. Uno es culpable de traición sólo si permanece en el lugar y subrepticiamente mina las instituciones del lugar mientras que en forma ostensible les muestra respeto y lealtad. (Hay todas las diferencias del mundo entre tal subversión subrepticia y la crítica abierta, por fuerte que sea, de las instituciones cívicas por considerarlas sin valor, obsoletas o cosas por el estilo)» (p. 170).

«Hay al menos una razón prima facie en favor de algún tipo de derecho a respetar las identidades nacionales como una parte del respeto hacia las personas, pues en el respeto hacia los individuos humanos está el respecto hacia aquello que forma parte de su individualidad. Los principios políticos que sean justificables moralmente tienen que dar cuenta de un sentido de la nacionalidad que es intrínseco a la formación de la individualidad, en la medida en que tal sentido es vital para muchos individuos para el sentido de ellos mismos y para su auto-respeto al ser considerados como miembros de una comunidad humana reconocida y respetada» (p. 183).

En todo momento subrayo que mientras que alguna forma de instituciones políticas representativas está justificada por referencia a pretensiones significativas de autodeterminación nacional, no puede ser que todos y cada uno puedan o deban llegar a ser Estados soberanos en el Derecho internacional; pero entonces, mi otra tesis es que la soberanía es parte del problema y no de la solución, y de ahí el título de mi libro.

M.A.: En estos días estoy leyendo tu libro Rhetoric and the Rule of Law. Me gusta mucho tu (relativamente) nueva orientación con respecto a Legal Reasoning and Legal Theory; como probablemente sabes, tu concepción del razonamiento jurídico tiene una gran influencia en la cultura de habla española. Estoy muy de acuerdo con los aspectos sustantivos de tu nuevo libro, pero no encuentro totalmente persuasivo el uso que propones de "retórica". En la página 7 afirmas que «el estudio de los buenos y malos argumentos se ha llevado a cabo, a lo largo de los años, bajo el nombre de "retórica"», pero creo que es una afirmación un tanto dudosa. Por ejemplo, los primeros parágrafos de la Retórica de ARISTÓTELES se consagran a clarificar la distinción (el paralelismo) entre retórica y dialéctica (de esta última se ocupa en la Tópica); en nuestros días los filósofos (los filósofos de ámbito general) interesados en la argumentación suelen partir de una distinción entre la aproximación lógica, dialéctica y retórica al tema; y yo diría que resulta bastante extraño calificar como "retórica" o como "una contribución a la retórica" teorías de la argumentación jurídica como las de DWORKIN o SUMMERS que — si te be entendido bien-están muy cerca de tu aproximación al tema. ¿No crees que quizás hayas ido demasiado lejos en tu homenaje a PERELMAN y a su nueva retórica? En mi opinión, el riesgo de tu propuesta reside en perder algunas diferencias interesantes (por ejemplo, entre la aproximación a la argumentación de TOULMIN y de PERELMAN) y también en subestimar los aspectos dialógicos o dialécticos ("dialéctica" y "diálogo" no están incluidos en el Índice de Materias de tu libro). ¿Qué piensas sobre ello?

N.M.: Tengo la incómoda sensación de que puedas tener razón sobre eso. Rhetoric and the Rule of Law era el título original de lo que ahora es el capítulo 2, e inicialmente concebí el libro como una colección de ensayos antes de decidirme a integrarlos en una forma (espero) más rigurosa. Pero todavía sigo encontrando el título atractivo. Ahora me doy cuenta de que puede ser un poco engañoso, al menos para algunos lectores. ¡Pero espero que el balance que hace del razonamiento jurídico sea mejor que el nombre del libro! 
M.A.: Has escrito dos libros y muchos artículos sobre el razonamiento jurídico. ¿Crees que bay todavía algún aspecto (importante) de ese campo que no hayas tratado aún? Si la respuesta fuera que sí, ¿por qué? Y, en general, ¿cuáles son, en tu opinión, los aspectos del razonamiento jurídico que necesitan todavía ser clarificados?

N.M.: No soy consciente de ninguna laguna manifiesta que tenga que ser cubierta. Me doy perfecta cuenta, sin embargo, de que otros llevarán mucho más lejos de lo que yo lo haya hecho la aplicación de la "inteligencia de las máquinas" a algunos de los problemas del razonamiento jurídico, y pudiera ser que con el tiempo ELLO transformará el razonamiento jurídico en formas que no podemos prever ni predecir.

Mi propia obra es más bien escasa en relación con la prueba y con el razonamiento acerca de hechos. Sobre estos temas, aprecio de manera especial el trabajo de William TwINING. De nuevo, creo que hay mucho trabajo de interés por delante y que se acelerará en relación con todos los tipos de prueba de alta tecnología (DNA, etc.) en los juicios civiles y penales.

Éstos son campos en los que hay mucho más trabajo por hacer y en relación con los cuales mi contribución ha sido comparativamente restringida. Dudo que yo tenga la energía o la capacidad para implicarme de manera estrecha en ese tipo de trabajo.

Hay más cosas que deben hacerse también en el "razonamiento práctico general" y sus aplicaciones al Derecho. ALEXY, DWORKIN y FinNIS son, en diferentes sentidos, líderes en eso. Yo espero hacer algún trabajo sobre esto en los próximos dos años.

M.A.: En tus últimas publicaciones pareces estar próximo a las ideas de DWORKIN en la teoría del Derecho y algo semejante puede decirse (pero desde hace ya muchos años) sobre tu afinidad con las ideas de ALEXY. Ahora bien, ellos dos son autores no positivistas en cuanto teóricos del Derecho. De abi mi pregunta: ¿sigues siendo un autor iuspositivista (un iuspositivista institucionalista)? ¿Qué piensas sobre la actual discusión sobre el positivismo jurídico (incluyente, excluyente, axiológico, etc.)

N.M.: En Institutions of Law: An Essay in Legal Theory (Oxford, 2007) declaro que mi posición es postpositivista. Hemos tenido mucho que aprender del positivismo analítico en aspectos que - creo- DWORKIN no ha sabido apreciar, pero el positivismo mismo es insostenible (bien sea duro, blando, incluyente, excluyente o axiológico). Desarrollo por extenso una argumentación en favor de esta posición en el capítulo 15 de Institutions, y expongo los desacuerdos que sigo teniendo con DwORKIN con respecto a la metodología en el capítulo 16. Hay también en este capítulo una extensa discusión acerca de la metodología analítico-interpretativa que aplico más detenidamente al desarrollar la teoría institucional del Derecho.

M.A.: Durante los últimos años has estado intensamente implicado en el desarrollo de la comunidad europea. ¿Cómo ves el futuro de Europa? ¿Crees que tendremos pronto una Constitución europea? ¿Tiene la teoría jurídica algún papel significativo que jugar en la construcción de Europa? ¿Cómo ves la actual situación de la teoría jurídica en Europa y en el mundo?

N.M.: Según mi punto de vista (expresado en Cuestioning Sovereignty, Oxford, 1999; y en Who's Afraid of a European Constitution?, Exeter: Imprint Academic, 2005) 
la Unión Europea tiene ya una Constitución en el "sentido funcional”. Está contenida en los tratados existentes, a los que la Corte de Justicia ha llamado «Carta Constitucional», y en los propios pronunciamientos de la Corte interpretando los tratados. Lo que falta es una Constitución formal, y la ventaja de adoptar una tal como la de la Convención sobre el futuro de Europa y adoptada por los Estados miembros en el Tratado de Roma de 2004 consistiría en salvar el déficit democrático y hacer el funcionamiento de la Unión más inteligible para sus ciudadanos. No puedo prever cuánto tiempo llevará que los Estados miembros se pongan de acuerdo para adoptar tal Constitución, pero seguramente ocurrirá durante los próximos diez años, espero que antes mejor que después. La aportación más importante de la teoría jurídica afecta a tomarse en serio la idea de que el Derecho de la Unión Europea es un «nuevo tipo de orden jurídico, sui generis». Si esto es así, tiene un especial interés para los teóricos. Mi propia contribución particular ha consistido en avanzar la teoría del pluralismo constitucional y, junto con ello, la idea de la postsoberanía. Creo que estas ideas pueden suponer una importante contribución en el actual debate jurídico-político.

La teoría jurídica en Europa y en todo el mundo es más excitante y más ampliamente debatida de lo que nunca lo había sido antes. Mi crítica es que ha tendido a convertirse en una teoría que mira más bien hacia adentro y contribuye menos de lo que debería al discurso general de los juristas, tanto en la academia como en la práctica. Por mi parte, siempre he procurado escribir de una manera que lo que pienso esté referido a problemas jurídicos reales, y recomiendo esta aproximación a otros cultivadores de la materia.

M.A.: Me gusta terminar este tipo de entrevistas pidiendo al entrevistado algún consejo para quienes están empezando su carrera en la filosofía jurídica. ¿Cuál sería tu mensaje para los jóvenes iusfilósofos? ¿Hay algún tema particular o algún tipo de aproximación que consideres de especial interés para ellos?

N.M.: Naturalmente, desearía que encontraran la teoría institucional del Derecho especialmente interesante, y considero Institutions of Law como la mejor exposición que puedo hacer de ella. Necesitamos olvidarnos de áridas disputas acerca de los diversos tipos de positivismos y mirar hacia todas las maneras en que las teorías valorativas tienen aplicaciones fructíferas a los problemas y dilemas jurídicos. No debemos confundir nuestro trabajo con el de la sociología del Derecho, pero deberíamos llevar a cabo nuestros estudios con un conocimiento mucho más profundo de los desarrollos en la sociología y en otras ciencias sociales de lo que ha solido ser el caso. La globalización es, y seguirá siendo, un gran problema y, junto con ello, el desarrollo del Derecho humanitario y la urgente necesidad de soluciones (en parte) jurídicas para los problemas del cambio climático.

(Trad. Manuel Atienza) 